\title{
Neutron clustering as a driver of Monte Carlo burn-up instability
}

\author{
P. Cosgrove, E. Shwageraus, G.T. Parks \\ Department of Engineering, University of Cambridge, Trumpington Street, Cambridge, CB2 1PZ, United \\ Kingdom
}

\begin{abstract}
Coupled Monte Carlo neutronics and depletion problems have been noted to produce nonphysical power oscillations for large, spatially-decoupled problems. Previously these oscillations have been attributed to 'numerical instability' - this work proposes that a prominent contributor to this phenomenon is neutron clustering during the Monte Carlo simulation, resulting in a poor estimate of the transport solution. This is demonstrated using insights from recent work on clustering and applying it to standard practice for Monte Carlo/depletion problems by performing simulations with the same number of histories - both total histories and only active histories - but different numbers of particles and cycles. The results demonstrate that neutron clustering appears to trigger instabilities in the problems considered and strongly affects Monte Carlo neutronics/depletion simulations.
\end{abstract}

Keywords: Depletion, Neutron transport, Monte Carlo, Clustering, Stability

\section{Introduction}

Monte Carlo neutron transport solvers are now ubiquitously used for high-fidelity reactor analysis. This usage is commonly extended to coupling with other physical phenomena such as isotopic depletion and thermal-hydraulics. However, Monte Carlo eigenvalue calculations also have their theoretical challenges, for example, ensuring source convergence (Ueki, 2008) or, more recently, the problem of neutron clustering (Dumonteil et al., 2014). This latter challenge is also related to noted difficulties with non-ideal convergence of Monte Carlo estimators (Brissenden and Garlick, 1986; Herman, 2014).

In coupling, Monte Carlo has also proved challenging: coupled neutron transport and thermal-hydraulics has been demonstrated as unstable, requiring slow relaxation and multiple transport/thermal-hydraulic solver iterations (Aufiero and Fratoni, 2017; Gill et al., 2017). Monte Carlo transport and depletion suffers from an apparently similar complication of 'instability': reports of non-physical behaviour in the form of flux/power/nuclide density oscillations are well-established, particularly for large, spatially-decoupled systems (Dufek and Hoogenboom, 2009; Dufek et al., 2013b; Kotlyar and Shwageraus, 2013; Isotalo et al.,

Email address: pmc55@cam.ac.uk (P. Cosgrove) 
2013; Kȩpisty and Cetnar, 2015; Josey, 2017). However, in spite of this instability, forcing xenon into equilibrium with the flux during the active cycles of a Monte Carlo simulation has proved beneficial in evading non-physical behaviour, as has aggressive relaxation with many iterations using the Stochastic Implicit Euler method (SIE) (Dufek et al., 2013a).

This paper postulates that the phenomena of neutron clustering and burn-up instability are linked: clustering occurs in precisely the same geometries where burn-up instabilities are observed, namely those in which the neutron migration length is small in proportion to the geometry's characteristic dimension length, i.e., geometries with a large dominance ratio. Clustering during the Monte Carlo simulation can result in a biased transport solution - for example, one which is noticeably asymmetric for an otherwise symmetric problem. This error in transport is then propagated to the depletion solver: explicit Euler solvers will magnify even small errors due to stochastic noise. However, Predictor-Corrector methods (PC) obtain a second transport solution at the End-of-Step (EOS) time-point in an attempt to correct the extrapolation. Unfortunately, the calculated eigenvector and resulting correction may also be contaminated by clustering.

This paper makes use of insights by Dumonteil et al. (2014) and Sutton and Mittal (2017) with respect to when clustering occurs and how it may be combated, and compares these suggestions with observed practice in coupled Monte Carlo/burn-up simulations. In particular, Dumonteil et al. (2014) and Sutton and Mittal (2017) suggest using large numbers of particles per cycle with relatively few cycles, in keeping with the work by Herman (2014), to reduce the influence of inter-cycle correlations. This is supported by simulating two PWR pin problems repeatedly, fixing the total number of histories but trading-off the number of particles per cycle against the number of cycles. The same experiments are repeated but fixing only the number of active histories. This is done to minimise the number of generations that are simulated: from the work of Sutton and Mittal (2017) it was demonstrated that simulating excessively many cycles can induce bias in a transport solution.

The authors make no pretence of utilising the mathematics of clustering to derive results about burn-up instability. Rather, given the state of knowledge on clustering, enough can be said to meaningfully apply this knowledge to the troublesome area of depletion instabilities. We believe this has emerged, in large part, as a symptom of neutron clustering, at least in the many cases detailed previously, as discussed in Section 3. However, arguably, if the authors removed all discussion of clustering, the results presented here would still stand and be interesting to the Monte Carlo user, although clustering appears to provide the correct framework in which to consider them.

The paper will proceed with a brief introduction to the basic theory behind Monte Carlo depletion schemes and neutron clustering. This is followed by a short survey of previous literature in which instability has been observed in coupled transport and depletion, comparing the statistics used against those recommended to avoid clustering. Finally, the above numerical experiments will be described in more detail, and their results presented and discussed. 


\section{Theory}

This section will describe the relevant aspects of depletion and Monte Carlo clustering theory.

\subsection{Monte Carlo neutronics \& isotopic depletion}

Monte Carlo neutronics and depletion have long been coupled together for depleting nuclear systems (Leppänen et al., 2015; Griesheimer et al., 2017; Isotalo, 2013; Kang and Mosteller, 1983; Kotlyar and Shwageraus, 2013; Poston and Trellue, 1999; Stankovskiy, 2012). The simplest of these coupling schemes is an explicit Euler scheme: from some initial nuclide density, a Monte Carlo transport solution is obtained, usually generating one-group microscopic reaction rates for reactions which are important to track during the depletion stage. These are calculated during the course of the simulation by integrating the product of the energy-dependent microscopic cross-sections, $\sigma(E)$, and scalar neutron flux, $\phi(E)$, in a given homogeneous region, denoted here by $V$, assuming some spatial discretisation of the problem such that:

$$
\langle\sigma, \phi\rangle=\int_{0}^{\infty} \mathrm{d} E \int_{V} \mathrm{~d}^{3} \boldsymbol{r} \sigma(E) \phi(\boldsymbol{r}, E)
$$

In reactor calculations the flux is usually normalised to a specified power level. A comparable procedure is used to calculate average fission yields. Combining this information with nuclide decay constants and branching ratios provides what is known as the burn-up matrix. That is, for a vector of nuclide densities, $\boldsymbol{N}$, in a given homogeneous region, the rate of change of these densities is given by the Bateman equation:

$$
\frac{\mathrm{d} \boldsymbol{N}}{\mathrm{d} t}=\mathbf{A}(\phi) \boldsymbol{N}
$$

where $\mathbf{A}(\phi)$ is the burn-up matrix with element $i, j$ describing the rate of production of the $i$-th nuclide from the $j$-th nuclide due to transmutation, fission, and decay (Bell and Glasstone, 1970). This matrix is also implicitly dependent upon the neutron flux, $\phi$, which in turn depends on the nuclide density. Thus, coupled neutronics and depletion is a non-linear problem, requiring some sophistication to obtain both a stable and accurate solution. Due to this non-linearity, when using the Monte Carlo method to obtain the transport solution for depletion, a statistical bias is induced, although it is typically small compared to the statistical uncertainty in the results (Dumonteil and Diop, 2011). Assuming a constant burn-up matrix, the formal solution of Eqn. (2) at some later time $t$, starting from $t_{0}$, is:

$$
\mathbf{N}(t)=\exp \left[\mathbf{A}(\phi) \cdot\left(t-t_{0}\right)\right] \mathbf{N}\left(t_{0}\right)
$$

making use of the concept of a matrix exponential. For the system of differential equations occurring in nuclear reactors, this matrix exponential solution can be accurately obtained using the Chebyshev Rational Approximation Method (Pusa, 2013). The explicit Euler scheme simply obtains a transport solution and its associated burn-up matrices, updates the 


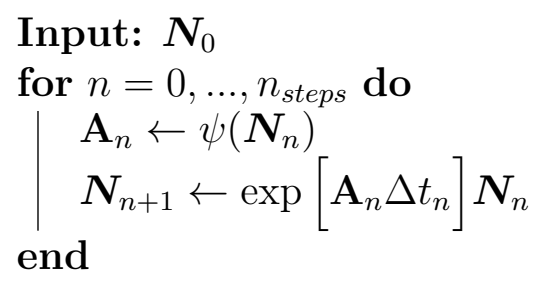

Algorithm 1: Explicit Euler method

nuclide densities in each homogeneous region using Eqn. (3) or an equivalent, and repeats for as many time-steps as required. The Euler scheme is shown in Algorithm 1.

Given that the burn-up matrix varies with the flux spectrum, explicit Euler depletion schemes are relatively rarely employed due to their inaccuracy. A more standard scheme is the PC method mentioned above. PC requires two transport solutions for a single time step: one at the Beginning-of-Step (BOS) which is used to burn the problem forward to the EOS, identically to the explicit Euler scheme. Another transport solution is obtained at this point, and an average of the region-wise reaction rates is calculated and used to burn the problem from the initial BOS to the subsequent BOS. This is shown in Algorithm 2.

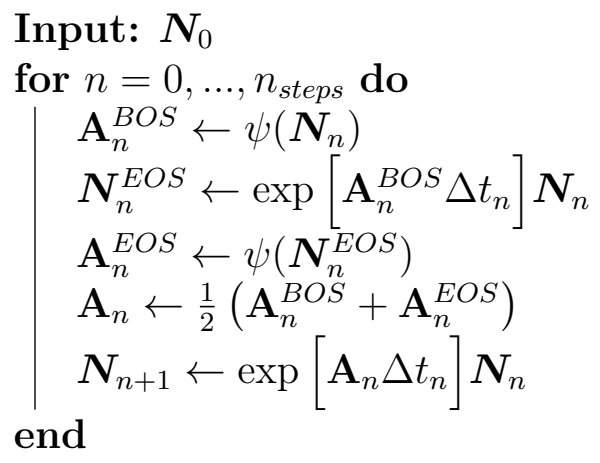

Algorithm 2: Predictor-Corrector method

Despite being more accurate than the Euler depletion method for the same length of time-step, PC has been shown to be numerically unstable (Kotlyar and Shwageraus, 2013; Dufek et al., 2013b). In particular, when considering large, spatially-decoupled burn-up problems, there is a tendency for calculated fluxes, reaction rates, and nuclide densities to oscillate in a non-physical manner with time. This behaviour is often observed when considering a geometry on the scale of a 3D fuel pin or larger and manifests with both reflective and vacuum boundaries axially. The SIE scheme mentioned in the introduction, along with its higher-order variants (Kotlyar and Shwageraus, 2014, 2016), was introduced as a 'numerically stable' coupling scheme. By iterating on the EOS transport calculation, SIE schemes perform an aggressive, iteration-dependent relaxation, either on the EOS nuclide density or reaction rates. If instability is attributable to clustering, then the SIE's success in obtaining stable solutions may be due partly to its EOS iteration: in the original paper it is shown that this iteration is equivalent to averaging a number of successively generated EOS reaction rates or nuclide densities. Although the simulations are not wholly independent, this 
averaging may smooth errors in the separate transport solutions resulting from clustering.

\subsection{Monte Carlo neutron clustering}

It has long been known that Monte Carlo methods tend to underestimate the standard deviation of responses, particularly in problems with large dominance ratios (Brissenden and Garlick, 1986; Brown, 2011). This is due to the correlation between consecutive generations of neutrons (Herman, 2014). The extreme case of this correlation occurs in the recently identified phenomenon of neutron clustering (Dumonteil et al., 2014): the combination of large problems in comparison to neutron migration lengths, neutrons being born adjacent to other neutrons, and population control due to the Monte Carlo algorithm results in particles in a simulation being clustered together, rather than distributed according to the eigenvector (Zoia et al., 2014; de Mulatier et al., 2015; Dumonteil et al., 2017). This has been shown to significantly affect the estimation of the eigenvector (Dumonteil et al., 2017).

The clustering phenomenon can be suppressed by simulating a sufficiently large number of particles per generation, $N_{0}$. A rough estimate for this number is given by Dumonteil et al. (2014) as:

$$
N_{0} \gg L^{3} / l^{3}
$$

Here $L$ is the characteristic size of the system and $l$ is the root-mean-square distance from a neutron's birth to its absorption. Dumonteil et al. (2014) thus estimate that one requires a number significantly in excess of 300,000 neutrons per generation to accurately model a $4 \mathrm{~m}$ tall PWR pin while avoiding clustering effects. Sutton and Mittal (2017) also suggest that simulating larger generations reduces the degree of clustering. The relation in Eqn. (4) implies that the severity of the clustering effect grows with the cube of the problem size. That said, a refinement to Eqn. (4) has been obtained by de Mulatier et al. (2015) for confined geometries with reflective boundaries, subject to neutron diffusion, stating rather that:

$$
N_{0} \gg L^{2} / \mathcal{M}^{2}
$$

Here $\mathcal{M}^{2}$ is the neutron migration area. For a PWR, the migration area is about $56 \mathrm{~cm}^{2}$, giving, for a $4 \mathrm{~m}$ pin, an $N_{0}$ on the order of 10,000. This particular scaling relation was investigated by Nowak et al. (2016) for a series of geometries, including a $4 \mathrm{~m}$ reflected PWR pin. It was shown that on the order of 500,000 particles per generation were adequate for modelling the problem. Regardless, for either of the relations presented, when increasing the height of, say, a fuel pin problem, increasing the number of particles simulated per generation (or the number of generations) to ensure a constant particle density per unit length is insufficient: clustering will occur eventually unless particle numbers increase superlinearly with the characteristic dimension. This point has been made by some of the authors mentioned above (and is demonstrated by Nowak et al. (2016)) but should be emphasised in the context of depletion: for some users, intuition might incorrectly suggest that a $4 \mathrm{~m}$ pin with 10 burnable regions might not even require as many particles as a 2D 17-by-17 PWR assembly, which will typically have many more burnable regions.

The work by Sutton and Mittal (2017) provides another important insight for clustering in Monte Carlo simulations: using excessively many particle generations can worsen the 
Table 1. Detailed descriptions of depletion studies

\begin{tabular}{|c|c|c|c|c|c|c|}
\hline Study & $\begin{array}{l}\text { Particles } \\
\text { per cycle }\end{array}$ & $\begin{array}{l}\text { Active } \\
\text { cycles }\end{array}$ & $\begin{array}{c}\text { Inactive } \\
\text { cycles }\end{array}$ & Geometry & Scheme & $\begin{array}{l}\text { Unstable } \\
\text { step (d) }\end{array}$ \\
\hline $\begin{array}{c}\text { Dufek and } \\
\text { Hoogenboom (2009) }\end{array}$ & 1,000 & 5,000 & 1,000 & $4 \mathrm{~m}$ pin, reflective & Euler & 2 \\
\hline Dufek et al. (2013b) & $\begin{array}{l}5,000 \& \\
300,000\end{array}$ & $\begin{array}{c}30,000 \& \\
400\end{array}$ & $\begin{array}{l}1,000 \& \\
500\end{array}$ & $\begin{array}{c}3 \mathrm{~m} \text { pin, reflective \& } \\
3.66 \mathrm{~m} \text { assembly, } \\
\text { vacuum }\end{array}$ & $\mathrm{PC}$ & $14 \& 5$ \\
\hline $\begin{array}{c}\text { Kotlyar and } \\
\text { Shwageraus (2013) }\end{array}$ & 300,000 & 1,200 & Unspecified & $\begin{array}{c}3.66 \mathrm{~m} \text { assembly, } \\
\text { vacuum }\end{array}$ & $\begin{array}{l}\text { Euler \& } \\
\text { PC }\end{array}$ & $5 \& 50$ \\
\hline Isotalo et al. (2013) & 5,000 & 5,000 & 1,000 & $4 \mathrm{~m}$ pin, reflective & $\mathrm{PC}$ & 1 \\
\hline $\begin{array}{l}\text { Kẹpisty and Cetnar } \\
\text { (2015) }\end{array}$ & 5,000 & 1,600 & 400 & $\begin{array}{c}3.66 \mathrm{~m} \text { assembly, } \\
\text { vacuum }\end{array}$ & Euler & 5 \\
\hline Josey (2017) & 32,000 & 2,500 & 2,500 & $\begin{array}{c}4 \mathrm{~m} \text { pin, reflective } \& \\
\text { vacuum }\end{array}$ & $\begin{array}{l}\text { Euler \& } \\
\text { PC }\end{array}$ & 7 \\
\hline
\end{tabular}

results obtained. This is a result of different neutron 'genealogies' dying off over generations such that it is possible, by the conclusion of a simulation, that all particles are descended from only one neutron in the initial source guess and are thus highly correlated spatially. While this 'fixation' is admittedly an extreme occurrence, it has the implication that arbitrarily many neutron generations should not be performed to obtain a more accurate solution. This is accounted for in Section 4.

\section{Survey of Monte Carlo depletion instabilities}

Monte Carlo depletion instabilities have been noted by numerous authors when using both the explicit Euler and PC schemes (or variants thereof). Table 1 details a number of these studies and the conditions under which flux oscillation or divergence was observed. All used radially reflective boundary conditions, with the axial boundary conditions specified under the Geometry heading. Likewise, the geometries used are either pins or assemblies. Each study also used more than 100 inactive cycles, and typically many more to assure source convergence and the stationarity of the Shannon entropy (Brown, 2011) - note that this does not account for the detrimental effects of excessive generations as described by Sutton and Mittal (2017). All studies describe PWR systems and so use similar power densities. The studies used as few as 8 and as many as 16 axial burnable regions - presently the difference in discretisation is not believed to strongly affect the nature of the instabilities. Coolant densities for each study were uniform except for the fuel assembly problems used by Dufek et al. (2013b) and Kotlyar and Shwageraus (2013): in these cases a realistic full power coolant density profile was used, decreasing with height as detailed in the papers.

Most authors make a point of declaring the arbitrariness of diagnosing instability, although this varies with problem complexity: for reflected problems with uniform coolant density, all burnable regions should be identical in flux and composition but will differ due to stochastic noise - the degree of non-uniformity which indicates instability is not welldefined, although qualitatively the instability often grows to become obvious when it occurs. While the coolant density remains uniform, applying vacuum boundaries axially means that each region is no longer identical; however, whatever solutions are obtained should be symmetric. Again, the degree of asymmetry which delimits noise from instability is not readily apparent. Finally, in the cases which consider a non-uniform coolant density, there is no obvious feature of the solution which can be exploited as an error measure: without either a 
physical or numerical benchmark, diagnosing these problems as definitively unstable is difficult, although dramatic changes in solution behaviour may be observed when using different time-step lengths. This is actually the reason why the unstable time-step length differs for the fuel assembly studies performed by Dufek et al. (2013b) and Kotlyar and Shwageraus (2013), with the former reporting 5 days and the latter reporting 50 days for an identical problem: oscillations can be observed with the smaller step, although their physicality is ambiguous, whereas for 50 day steps there is a notable divergence in the calculated eigenvalue as compared to steps of 25 days or less. Although the current paper proposes neutron clustering as a confounding factor in Monte Carlo/depletion problems, it does not rule out the existence of true numerical instabilities driven by time-step length, which may well be occurring in the case of Kotlyar and Shwageraus (2013) and which might afflict deterministic transport/depletion simulations also. This has been demonstrated for a very simple deterministic case by Densmore et al. (2013).

In (Dumonteil et al., 2014), Fig. 2 strikingly presents the neutron distribution over cycles for a fully-reflected $4 \mathrm{~m}$ tall pin when using 10,000 neutrons per cycle. This simulation clearly fails to reach the eigenvector, but, even so, some of the studies above have used fewer particles per generation while attributing non-physical solutions to 'numerical instability'. Furthermore, none of the studies above used sufficiently many particles per cycle to avoid clustering according to the recommendations by Dumonteil et al. (2014), and only Kotlyar and Shwageraus (2013) and the second study by Dufek et al. (2013b) used nearly as many particles as Nowak et al. (2016) found satisfactory for a reflected pin $(500,000)$ - indeed even this may not be adequate for their cases with a vacuum boundary. However, this could be done while conserving statistics by trading-off between particles per cycle and the number of active cycles: if clustering is causing the instabilities, then simulating fewer, larger cycles should be noticeably more effective than simulating many small cycles (provided source convergence has been achieved during the inactive cycles).

\section{Numerical experiments}

This paper investigates the effect of neutron clustering on coupled Monte Carlo and depletion by performing burn-up simulation on two sets of standard PWR pins: one with a uniform coolant density, the other with a coolant density corresponding to that used by Kotlyar and Shwageraus (2013), and both with an initially uniform fuel composition. This former problem was chosen because its solution must be symmetric, making it easier to identify when a given solution becomes non-physical. It is also a standard problem when considering Monte Carlo burn-up instabilities as seen from Table 1.

The latter problem's asymmetry does not allow for the assertion of any property that a stable solution must possess, making its behaviour difficult to distinguish from numerical artefacts. However, it is worth considering in order to confirm or exclude the effects of clustering on more realistic problems and determine the prominence of true numerical instability. This can be attempted qualitatively by noting large differences in the eigenvectors where none should otherwise be expected, i.e., between simulations using different numbers of particles but the same number of histories. 
It should also be noted that only low-order depletion schemes are used here, as opposed to sub-stepping methods (Isotalo and Aarnio, 2011). The accuracy of depletion is not of concern in this work beyond the general behaviour of the solutions. Indeed, higher-order methods may have different stability properties compared to their one-step variants, although this is not considered presently.

The pin is $3.66 \mathrm{~m}$ tall, with a pitch of $1.26 \mathrm{~cm}$, and is surrounded axially by $20 \mathrm{~cm}$ thick homogenised steel/water reflectors. The fuel is $5 \%$ enriched uranium dioxide of radius $0.4095 \mathrm{~cm}$, clad with zirconium of $0.0655 \mathrm{~cm}$ thickness. The fuel is axially discretised into 10 burnable regions and burned at a constant power of $60 \mathrm{~kW}$, corresponding to a typical PWR power density of $104 \mathrm{~W} / \mathrm{cm}^{3}$. The boundary conditions applied are radially reflective and axially black. Both the Euler and PC algorithms are used with maximum time-step lengths of 20 and 40 days.

Two comparisons of simulation parameters will be made: one in which the number of particles per generation is varied while the total number of histories simulated is kept constant, the other in which the particles per generation are varied while only the active histories are kept constant. The logic behind preserving only the active histories follows from the point in Section 2.2: Sutton and Mittal (2017) suggest that simulating excessively many generations of particles can further bias the solution. If, as is often found in practice, fission source convergence is achieved in relatively few generations and independent of the number of particles per generation, regardless of the number of particles simulated, then simulating additional generations only serves to penalise simulations using fewer particles per generation. Both sets of results will be presented. In both cases, if burn-up instability is a problem purely of statistics, this investigation would have no effect on its likelihood of occurrence due to the active histories remaining the same for all simulations. On the other hand, if clustering is a driver, then more particles per cycle with fewer active cycles should suppress instability more successfully than many active cycles with relatively few particles. Each permutation of the simulations (maximum time-step, neutrons/cycle, depletion scheme, etc.) is run three times with different seeds to better account for variations driven by noise.

All simulations are performed using the Monte Carlo code Serpent v.2.1.30 which has integrated depletion capabilities (Leppänen et al., 2015). Although it is possible to initiate a transport solution using the fission source from the previous time-step, this was not done in the present work. That said, if clustering is occurring to any significant extent, passing the possibly biased fission source from one time-step to the next may amplify the resulting error over time-steps. Version 2.1.30 was modified to produce the first moment of the Shannon entropy. The variation and amplitude of the first moment of the entropy has been demonstrated as indicative of the degree of neutron clustering (Nowak et al., 2016). As an illustration of the degree of clustering in different simulations, for the first time-point for the uniform coolant problem, three different simulations using different numbers of particles will have their entropy moment presented as far as they overlap.

For the pin with uniform coolant density, the degree of instability was quantified by 
considering the spatial asymmetry of the flux, calculated as:

$$
\epsilon=\sum_{i=1}^{5} \frac{\left|\phi_{i}-\phi_{10-i+1}\right|}{\phi_{\max }}
$$

where $\phi_{i}$ is the flux in homogeneous region $i$, and $\phi_{\max }$ is the maximum value of flux in the geometry. The variable coolant density problem has no obvious error measure to exploit. Instead, one 3,000,000 particle simulation will be used as a reference, for a given time-step length and depletion scheme, and a normalised $L_{1}$ error in flux will be computed for each time-point:

$$
\epsilon=\sum_{i=1}^{10} \frac{\left|\phi_{i}-\phi_{i}^{*}\right|}{\phi_{\max }^{*}}
$$

where $\phi^{*}$ is the chosen reference solution. To provide further context to the non-uniform coolant density results, the flux distributions at the end of each simulation are also plotted. To emphasise: no solution can be definitively chosen as a reference, but this measure should identify differences between solutions where they would not otherwise be anticipated. Furthermore, it should also be emphasised that Eqns. (6) and (7) are entirely unrelated to clustering and inappropriate for measuring its extent (unless the clustering is very extreme). Instead, they are purely used as a measure of the error in transport solutions through depletion.

\section{Results and discussion}

\subsection{Uniform coolant density}

The flux asymmetries for the Euler and PC schemes with 20 day maximum steps are shown in Figs. 1 and 2 respectively. Each figure shows the results of running 30,000, 300,000, and 3,000,000 particles per generation over three different seeds - colour is used to identify the number of particles per generation, the different line styles delineate different seeds. Each figure features the results when preserving the total number of histories and when preserving the total number of active histories. In the former case, the number of active/inactive cycles for 30,000, 300,000 and 3,000,000 particles are 5,000/10,000,500/1,000, and 50/100, respectively. For the latter, the settings are 5,000/100, 500/100, and 50/100 (the last set of simulations, the 3,000,000 cases, are identical in each sub-figure). Given the statistics applied, in all cases the maximum uncertainty in the flux estimates according to Serpent was on the order of $0.1 \%$ of their nominal value.

The Shannon entropy was considered and it was found that, for the first time-point, 100 inactive cycles was adequate to converge the fission source (although it was markedly more jagged for simulations using fewer particles, indicative of clustering) - when instability has not occurred, source convergence occurs more rapidly as the fission source profile is flattened by symmetrically distributed fission products. Arguably, where instability occurs, 100 inactive cycles is insufficient to converge the source profile due to the asymmetry that develops. However, this provides a degree of conservatism - as fission sources are not passed 

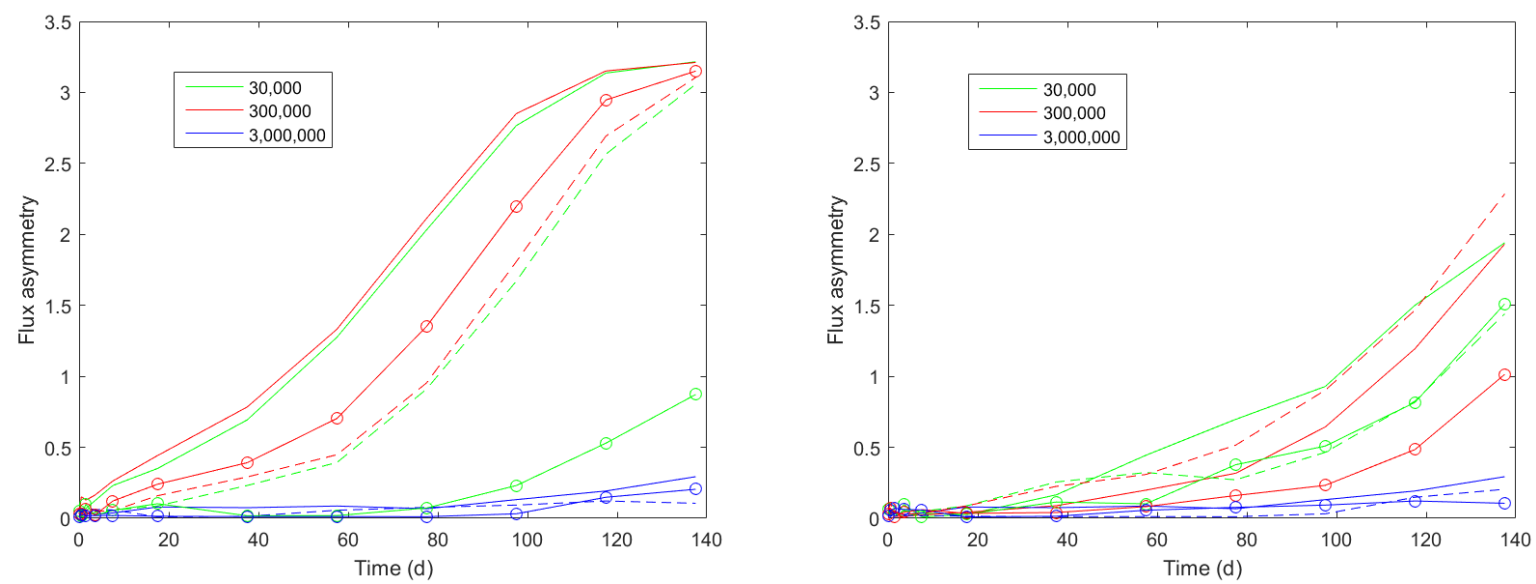

Fig. 1. Flux asymmetry with time, conserving total histories (left) and active histories (right) when depleting a PWR pin with uniform coolant density using the Euler scheme, with a 20 day maximum time-step, and varying the number of particles per generation
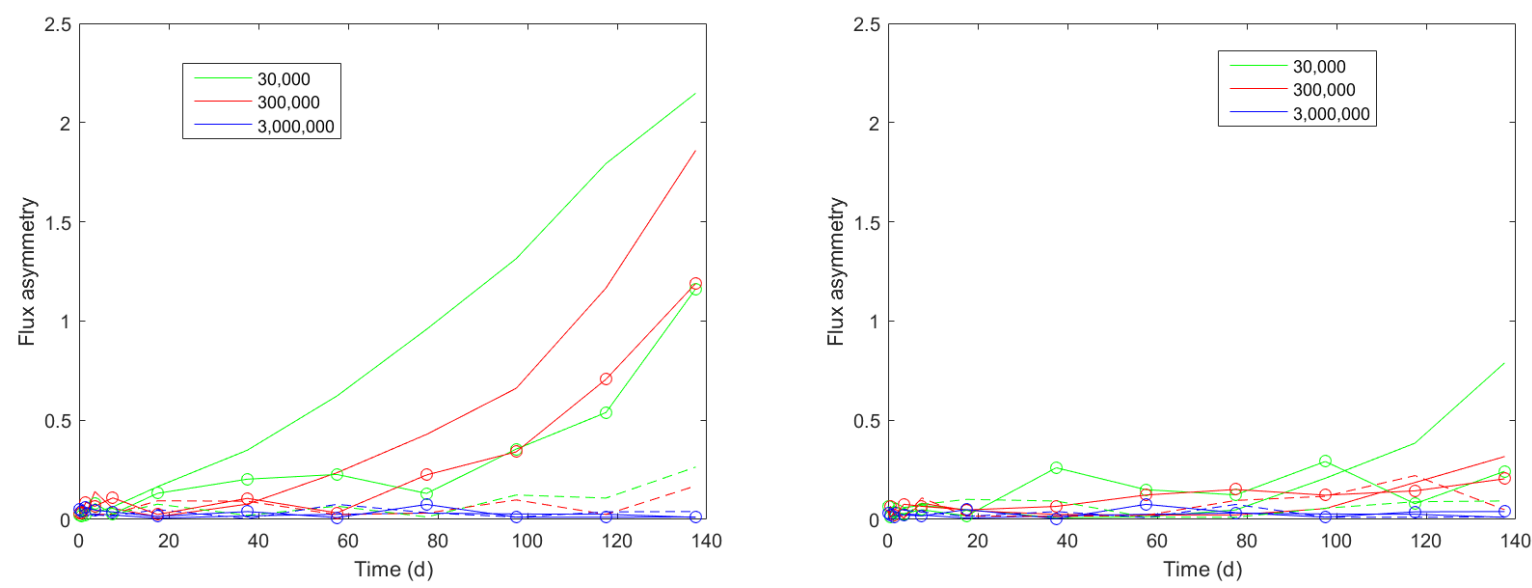

Fig. 2. Flux asymmetry with time, conserving total histories (left) and active histories (right) when depleting a PWR pin with uniform coolant density using the PC scheme, with a 20 day maximum time-step, and varying the number of particles per generation 


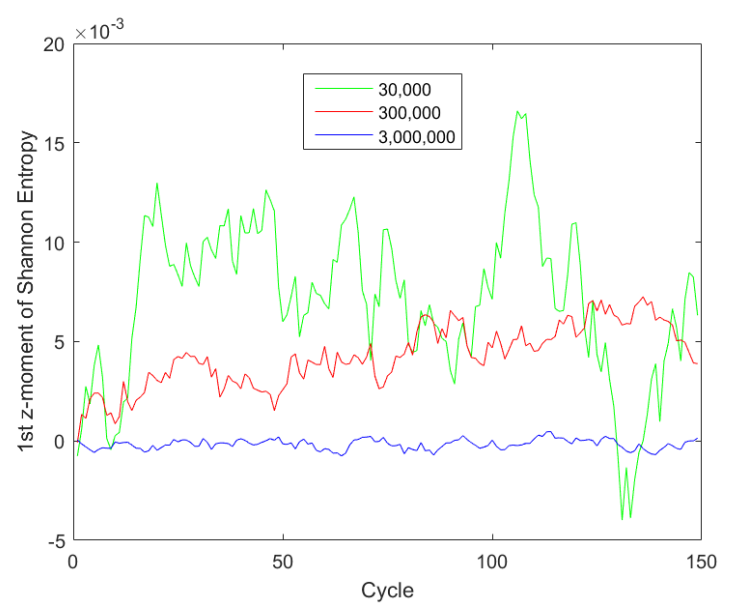

Fig. 3. The first z-moment of the Shannon entropy for the first 150 cycles of a PWR pin with uniform coolant density when varying the number of particles per generation

from one time-step to another, a flat, symmetric profile is initially assumed by Serpent, biasing any asymmetric problem towards symmetry. To briefly demonstrate the relative degree of clustering, Fig. 3 shows the first moment of Shannon entropy for three simulations in Fig. 1 - as this value should be centred about zero in symmetric problems and have a zero amplitude, this demonstrates, as expected, that using fewer particles per generation results in a greater degree of clustering.

The first aspect of both Figs. 1 and 2 to be highlighted is that for both 30,000 and 300,000 particles per generation, when simulating the same total number of histories, extreme differences in behaviour can be observed between different seeds. For example, with the Euler scheme, asymmetry is seen to develop either immediately and noticeably by 20-40 days or may take longer to trigger, in the one case which develops at 100 days. Likewise, for the PC scheme, asymmetry is either immediate and dramatic or gradual to the point of arguably not occurring in the case of the dashed lines in Fig. 2. On the other hand, asymmetry growth occurs much more slowly when only preserving the number of active histories between simulations but still occurs noticeably for both the Euler and PC schemes. This is in agreement with the predictions of Sutton and Mittal (2017) regarding the exacerbation of clustering by simulating excessively many generations - even in problems which are not as pathological as that described in their original paper.

While the fewer particles per generation cases exhibit significant variation in behaviour, 3,000,000 particle simulations appear to perform well across all realisations with a uniformly small flux asymmetry. That being said, some stochastic noise is noticeable in the PC case, while a mild growth in asymmetry appears to occur at about 100 days in the Euler case. It is difficult to say what the cause of this latter phenomenon is definitively. A possible explanation is that true numerical instability is occurring: 20 day time-steps may be unstable, exciting higher solution modes, albeit more slowly than simulations with suspected clustering. This is surmised given the gentle gradient of asymmetry growth of 3,000,000 particle simulations as compared to those with fewer particles in Fig. 1. Alternatively, the 
Euler scheme may be more strongly susceptible to stochastic noise and this manifests as an error accumulation across time-steps.

It should also be highlighted that - whether using the same total histories or only active histories between simulations - there are no significant differences between the 30,000 and 300,000 particle simulations: whether the asymmetry grows immediately or at a later stage, a greater or lesser degree of clustering appears to have little effect. The asymmetry growth of some simulations is even strikingly similar in the left plot of Fig. 1, where the dashed and unbroken lines for the 30,000 and 300,000 cases track each other remarkably, entirely by coincidence, and even use different seeds. In fact, asymmetric solutions appear to be evolving to the same state, based on the convergence of the lines to the same asymmetry value in Fig. 1 - inspecting the end-of-burn-up flux profiles of the most asymmetric cases reveals they are all quite similar, the main difference being whether or not they are in-phase with each other. Clustering seemingly must be avoided nearly entirely, as with the 3,000,000 particle cases, in order to prevent instabilities.

The results for 40 day time-steps are presented in Figs. 4 and 5. For both schemes, the behaviour is broadly consistent with the 20 day time-step cases. There are two points to note, however.

The first is that the Euler scheme exhibits interesting behaviour across the three $3,000,000$ particle seeds: one of the seeds (the blue circled line) shows an early increase in flux asymmetry as compared to the others, and even compared to one of the 300,000 particle seeds initially. This might be attributable to the combination of some small noise occurring unavoidably (in this case, by about the 20 day time-point) and it being magnified by true numerical instability due to the relatively large time-step. This is supported by the asymmetry growth for this seed being much slower than that of the 300,000 particle case, which is initially more symmetric but more quickly reaches an asymmetric state, as well as the growth in asymmetry of the 3,000,000 particle cases, with each occurring at approximately the same rate. Regardless, given the degree of asymmetry, when using the Euler scheme for the uniform coolant density pin problem, avoiding clustering is not sufficient to assure a physical solution. On the other hand, the PC scheme performs well when using 3,000,000 particles, although with a marginally greater asymmetry than in the 20 day time-step cases. The 3,000,000 particle cases ultimately produce the only physically reasonable solutions, regardless of how many inactive cycles are used.

The second point is that, in the cases preserving the number of active histories, the 300,000 particle per generation simulations appear to suffer from instability less severely than the 30,000 particle cases in that, for the most part, their asymmetry growth tends to occur at a later point in time. One might anticipate this given that a lesser degree of clustering is to be expected when using more particles per generation, although this may also be purely chance through the random number seeds applied; definitively demonstrating the relative stability of the 300,000 particle simulations would require many more simulations.

\subsection{Non-uniform coolant density}

When considering the problem with non-uniform coolant density, all particles statistics were kept the same as in the uniform case except for the number of inactive cycles applied 

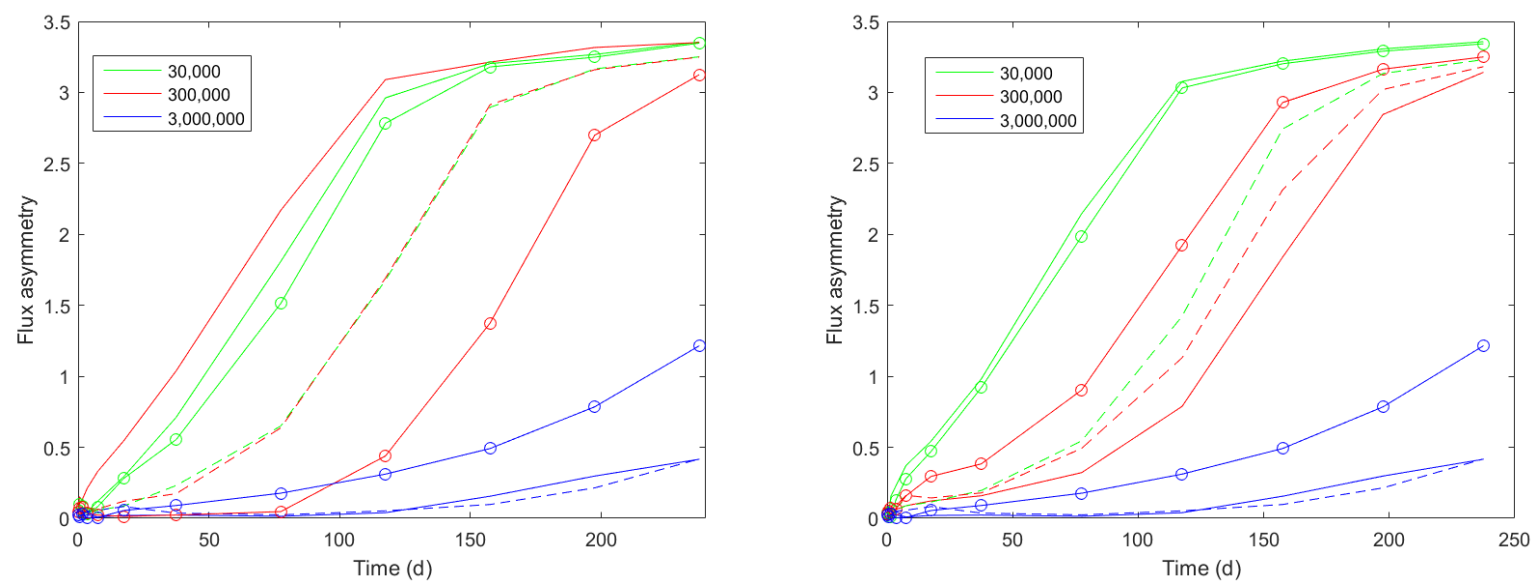

Fig. 4. Flux asymmetry with time, conserving total histories (left) and active histories (right) when depleting a PWR pin with uniform coolant density using the Euler scheme, with a 40 day maximum time-step, and varying the number of particles per generation
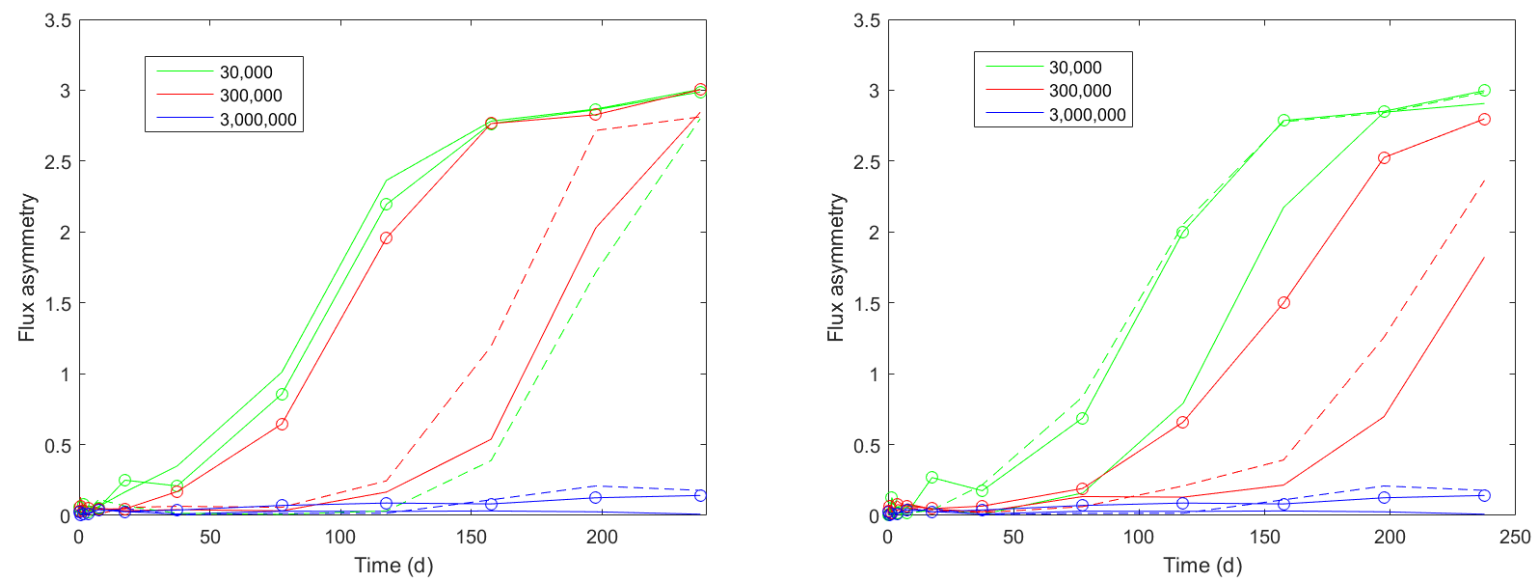

Fig. 5. Flux asymmetry with time, conserving total histories (left) and active histories (right) when depleting a PWR pin with uniform coolant density using the PC scheme, with a 40 day maximum time-step, and varying the number of particles per generation 
to the 3,000,000 particles per generation case and the cases preserving active histories only: this was increased from 100 to 200. This was done because the unburned fuel's Shannon entropy was not adequately converged in 100 cycles, although it was for subsequent timepoints due to the effects of fuel depletion making the fission source more uniform in the problem, better corresponding to Serpent's initial uniform fission source guess. Nonetheless, this does not appear to have affected the results.

For the 20 day time-step cases, the Euler $L_{1}$ errors and final flux distributions when preserving the total number of histories and the active histories are shown in Figs. 6 and 7, respectively. The same for the PC scheme are shown in Figs. 8 and 9, respectively. The colour and style of the lines on the flux error graphs correspond to the matching line on the flux profile graphs - this excludes the blue dashed line which is the reference solution used to compute $L_{1}$ errors and so only appears in the flux profile graphs.

Considering first the Euler scheme, the flux error behaviour suggests that using more particles delays the onset of instability but does not seem to prevent it, as seen by the convergence in $L_{1}$ error and the similarity of the final fluxes. What appears to be the case is that the highly-peaked, non-physical but 'stable' state manages to capture each simulation but at different times, depending on noise. There is one case when running 3,000,000 particles which displays a quick growth in error (the blue line with circles). However, considering the corresponding final flux profile, it actually appears not to be so greatly in error - rather, the other two 3,000,000 particle cases began to converge to the alternative, 'attracting state' more quickly, while the third moved to the non-physical solution only more gradually.

Ultimately, the behaviour here differs markedly from the uniform coolant density problem where the Euler scheme with 20 day time-steps remained physically reasonable when using large numbers of particles. Here, all simulations transition from a physical solution to a nonphysical one, albeit with a slightly longer delay when using more particles. When preserving only active histories this also hold true between the 30,000 and 300,000 particle simulations: considering Fig. 7, the simulations with fewer particles fall into error more rapidly, resulting in the $L_{1}$ error lines for different numbers of particles grouping together.

The PC scheme is more successful, although still strongly affected by clustering: from Fig. 8, the $L_{1}$ errors of the 3,000,000 cases remain small in comparison to the cases using fewer particles. Furthermore, the final fluxes have all apparently diverged to a different distribution when using fewer particles. Hence, clustering must be considered in depletion even where there is a realistic degree of asymmetry in the problem. This applies when preserving either total histories or only active histories, albeit, again, the error in the 300,000 particle cases grows more slowly when preserving active histories.

Figs. 10, 11, 12 and 13 show the behaviour of the same problem with a 40 day maximum time-step instead. Here the behaviour of both the Euler and PC schemes are similar: initially the simulations with fewer particles diverge from the 3,000,000 particle cases quickly, even before the time-steps have increased to their maximum length. However, eventually all cases, regardless of the number of particles per generation, converge towards the same solution.

There are two possible explanations. The first is that, although clustering may not be occurring for the 3,000,000 particle simulations, stochastic noise is still present, even if relatively small in amplitude. Nonetheless, it may be sufficiently large to eventually excite 

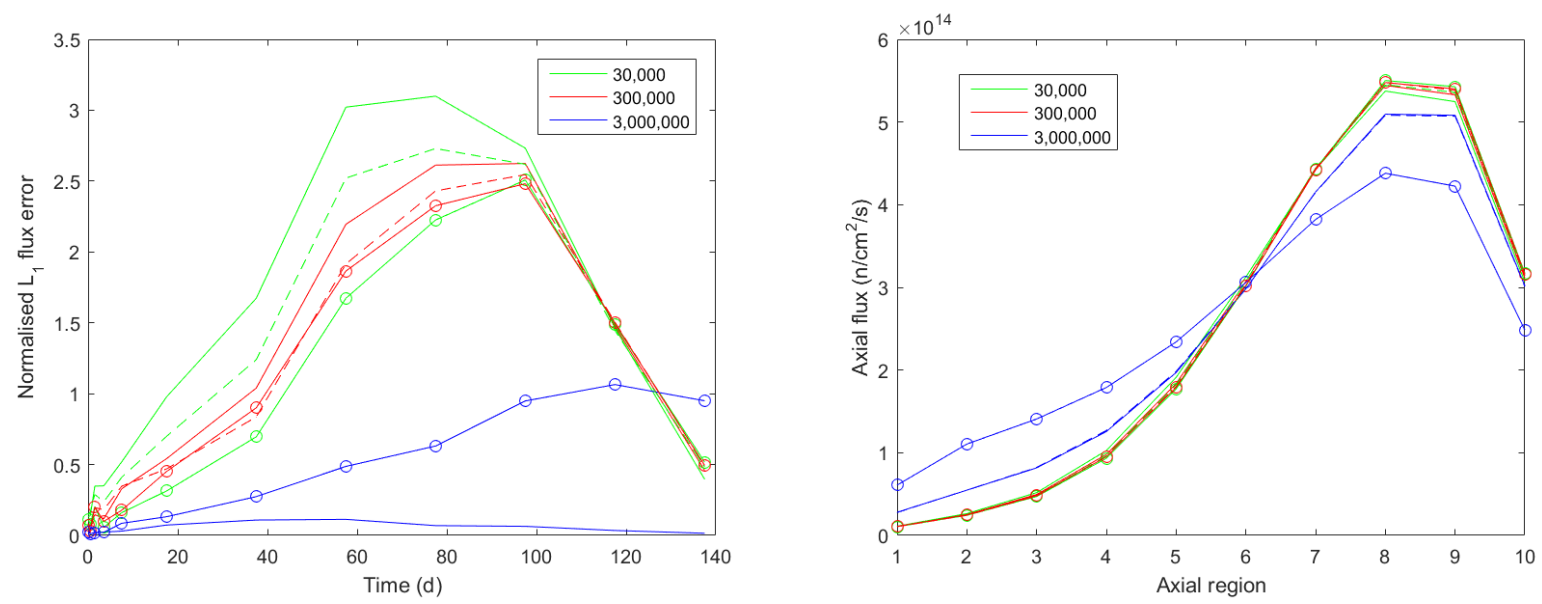

Fig. 6. Relative flux errors with time and final flux profiles when depleting a PWR pin with non-uniform coolant density using the Euler scheme, with a 20 day maximum time-step, and varying the number of particles per generation while preserving the total histories
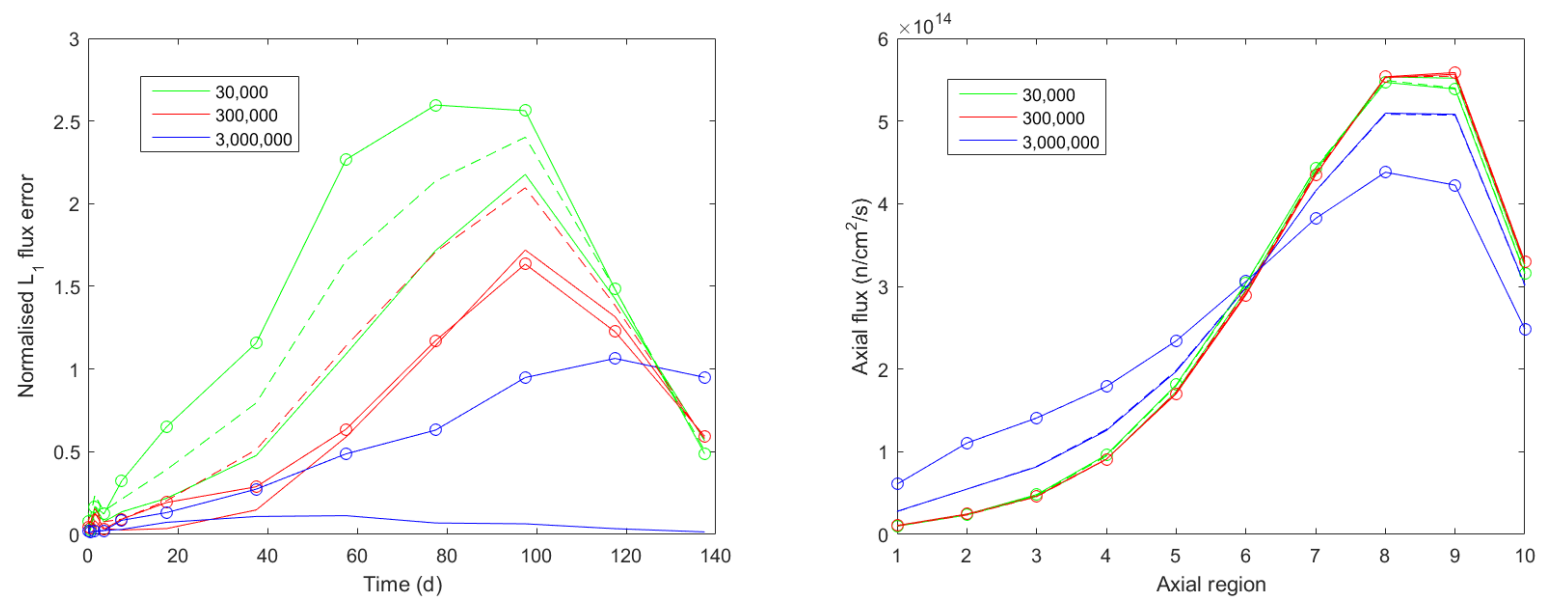

Fig. 7. Relative flux errors with time and final flux profiles when depleting a PWR pin with non-uniform coolant density using the Euler scheme, with a 20 day maximum time-step, and varying the number of particles per generation while preserving the active histories 

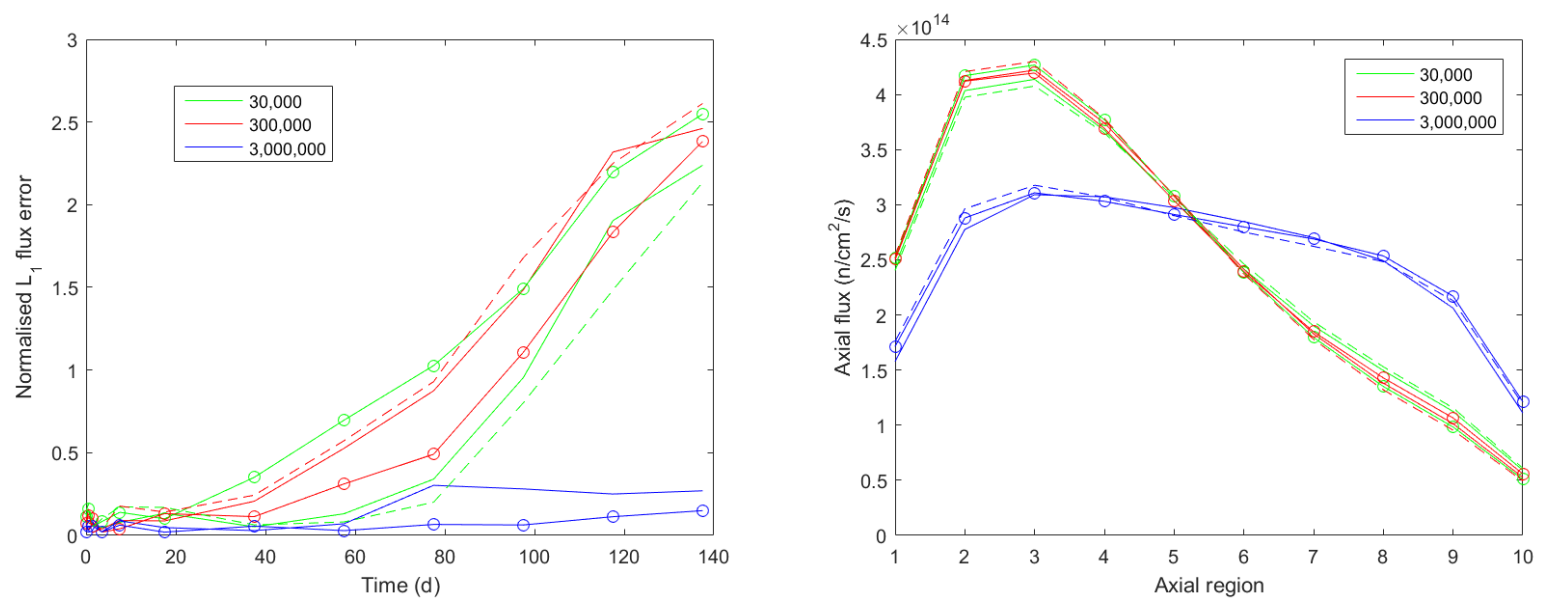

Fig. 8. Relative flux errors with time and final flux profiles when depleting a PWR pin with non-uniform coolant density using the $\mathrm{PC}$ scheme, with a 20 day maximum time-step, and varying the number of particles per generation while preserving the total histories
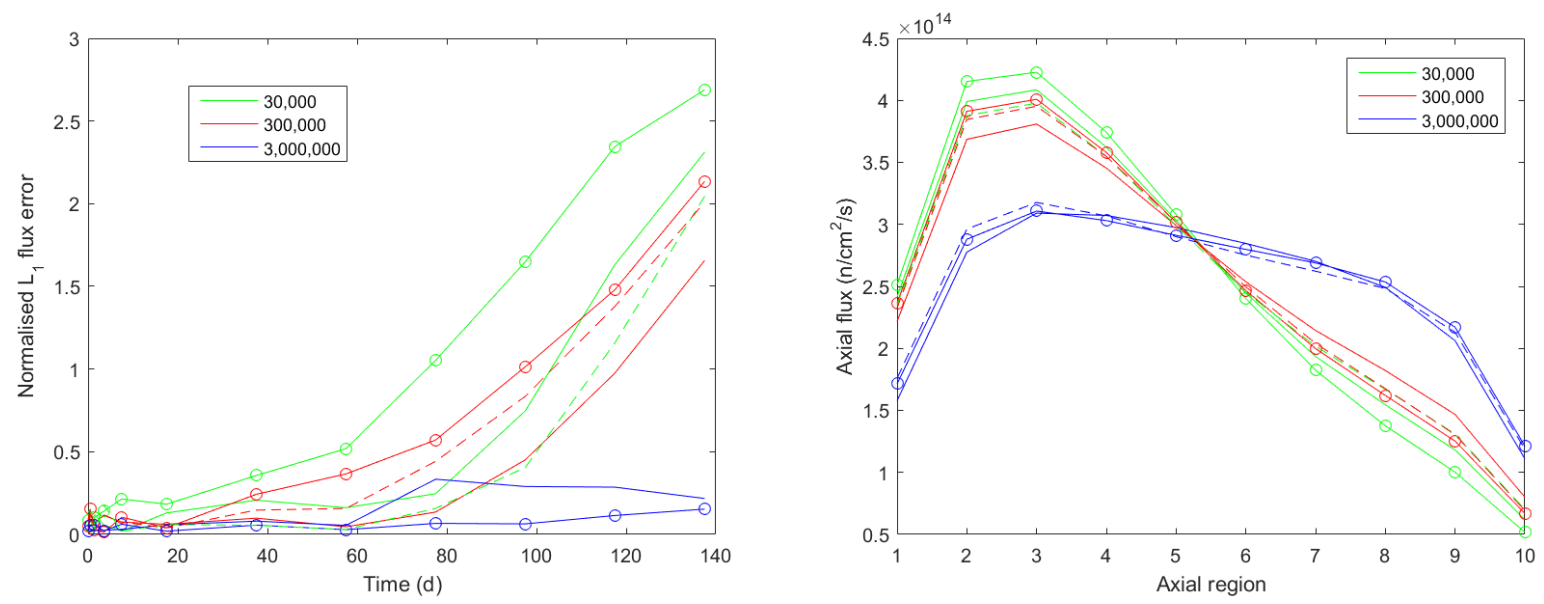

Fig. 9. Relative flux errors with time and final flux profiles when depleting a PWR pin with non-uniform coolant density using the $\mathrm{PC}$ scheme, with a 20 day maximum time-step, and varying the number of particles per generation while preserving the active histories 

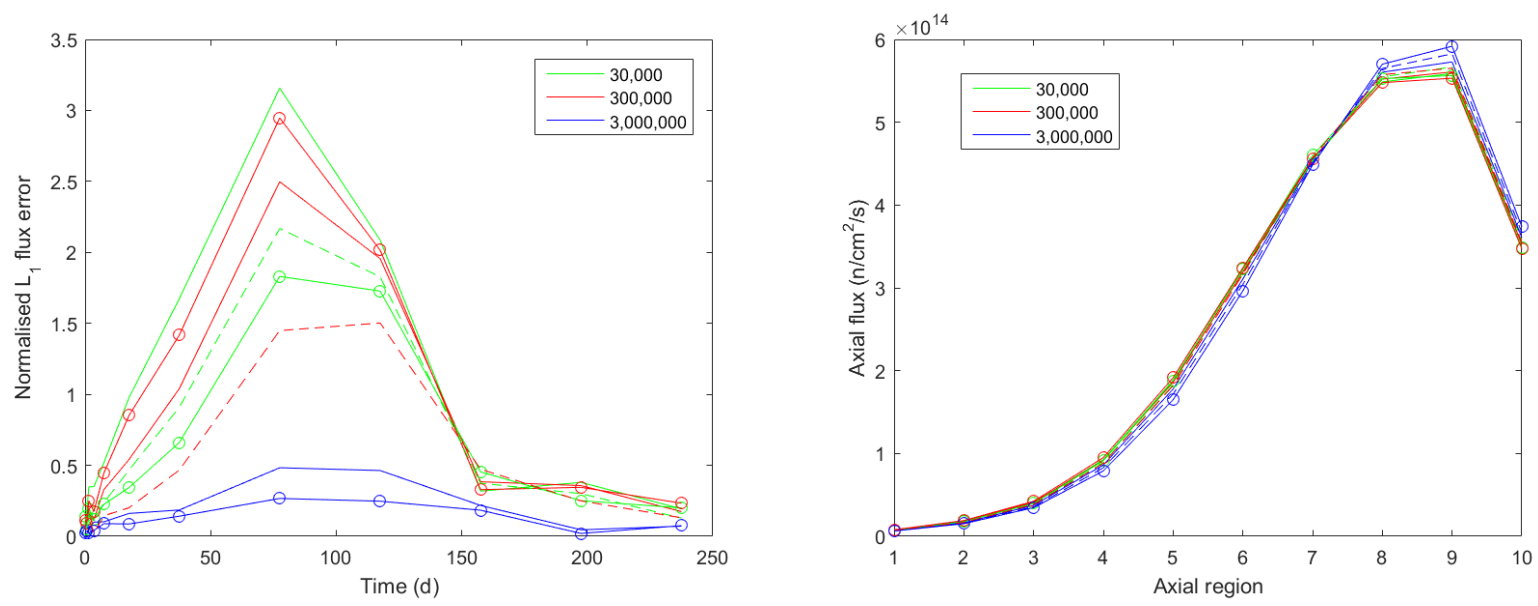

Fig. 10. Relative flux errors with time and final flux profiles when depleting a PWR pin with non-uniform coolant density using the Euler scheme, with a 40 day maximum time-step, and varying the number of particles per generation while preserving the total histories

the other 'stable' solution to which all simulations converge. Alternatively, true numerical instability driven purely by the time-step length may be occurring, as suggested by Kotlyar and Shwageraus (2013) and Densmore et al. (2013): for the given problem and for both schemes, the time-step taken may be sufficiently large that a non-physical solution would eventually be excited, regardless of the magnitude of the noise present. Densmore et al. (2013) demonstrated this for a simple deterministic problem where the only noise present resulted from finite precision arithmetic. If this is the case, the presence of stochastic noise may result in the alternative solution dominating more quickly, but the result would be the same whether or not it was present. This would also explain the behaviour of the Euler scheme in the uniform coolant density problem with 40 day time-steps and the non-uniform problem with 20 day time-steps: both of these problems are numerically unstable for the Euler scheme, whereas the PC scheme is relatively robust, only becoming unstable when applied to the non-uniform problem with a time-step of 40 days.

\subsection{A remark on the number of particles to avoid non-physical results}

By the estimate of Eqn. (5), simulating many more than 10,000 particles per cycle should be adequate to avoid clustering for a PWR pin. Less optimistic is the number of particles simulated by Nowak et al. (2016) for a reflected pin - 500,000 - which seems to avoid clustering. This does not appear to be the case here, or, at least, 300,000 particles are inadequate across the simulations performed in this work as regards preventing instability. That being said, Eqn. (5) was derived for a confined geometry with reflecting boundaries, assuming neutron diffusion (de Mulatier et al., 2015) while Nowak et al. (2016) were considering a reflected problem rather than the problem with axially vacuum boundaries as considered here. There are two possibilities to help explain this discrepancy: the first is the use of vacuum boundaries may serve to increase the necessary number of neutrons to simulate. This is briefly discussed in (Nowak et al., 2016) as an explanation for a factor of 

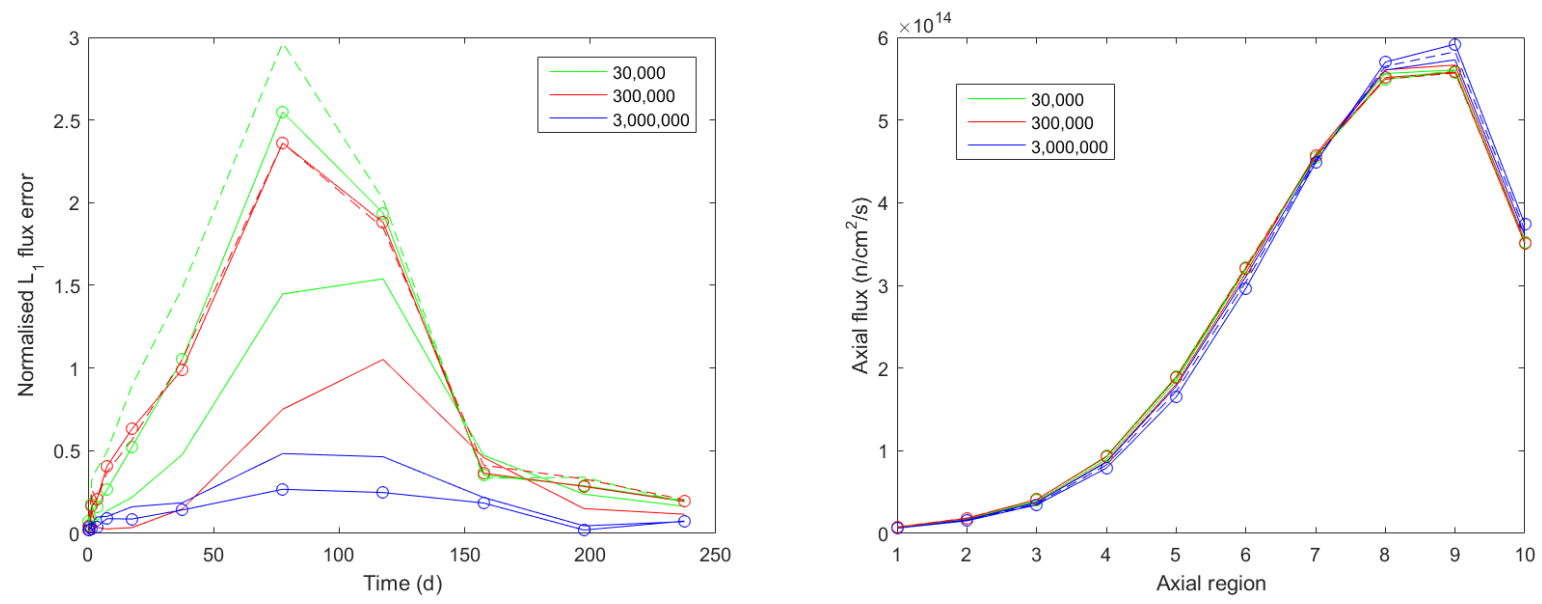

Fig. 11. Relative flux errors with time and final flux profiles when depleting a PWR pin with non-uniform coolant density using the Euler scheme, with a 40 day maximum time-step, and varying the number of particles per generation while preserving the active histories
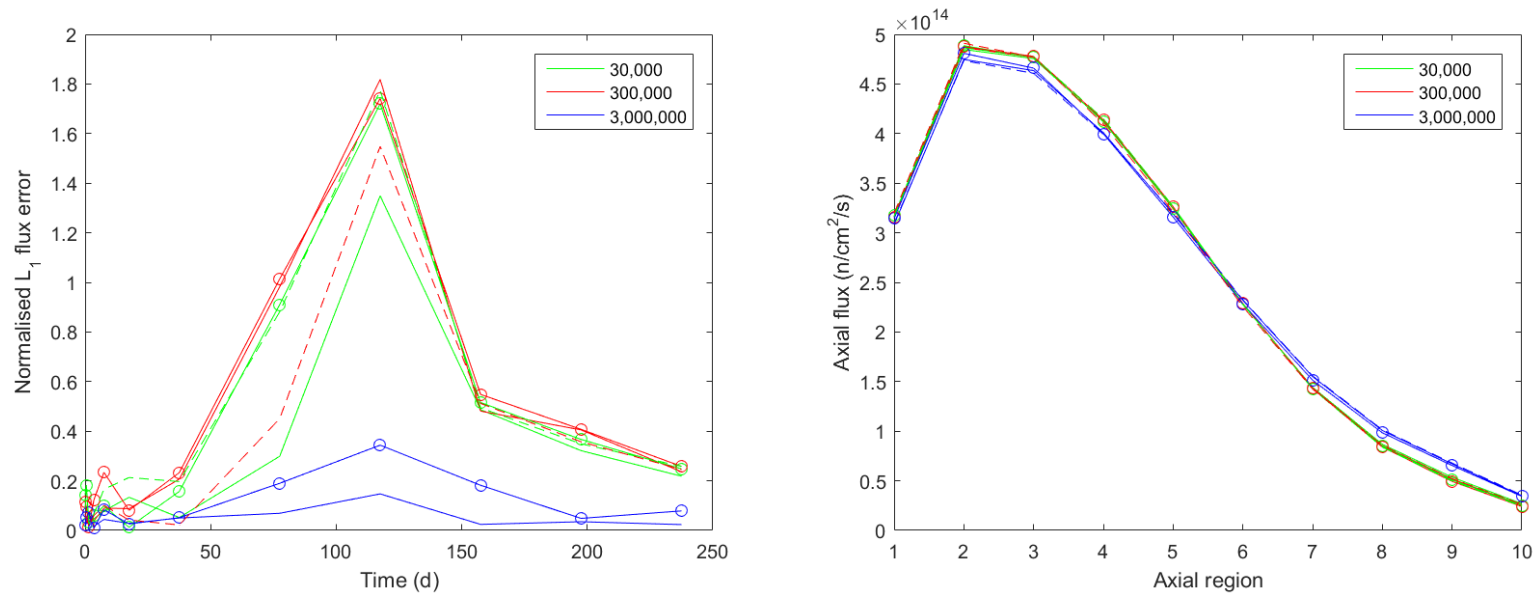

Fig. 12. Relative flux errors with time and final flux profiles when depleting a PWR pin with non-uniform coolant density using the PC scheme, with a 40 day maximum time-step, and varying the number of particles per generation while preserving the total histories 

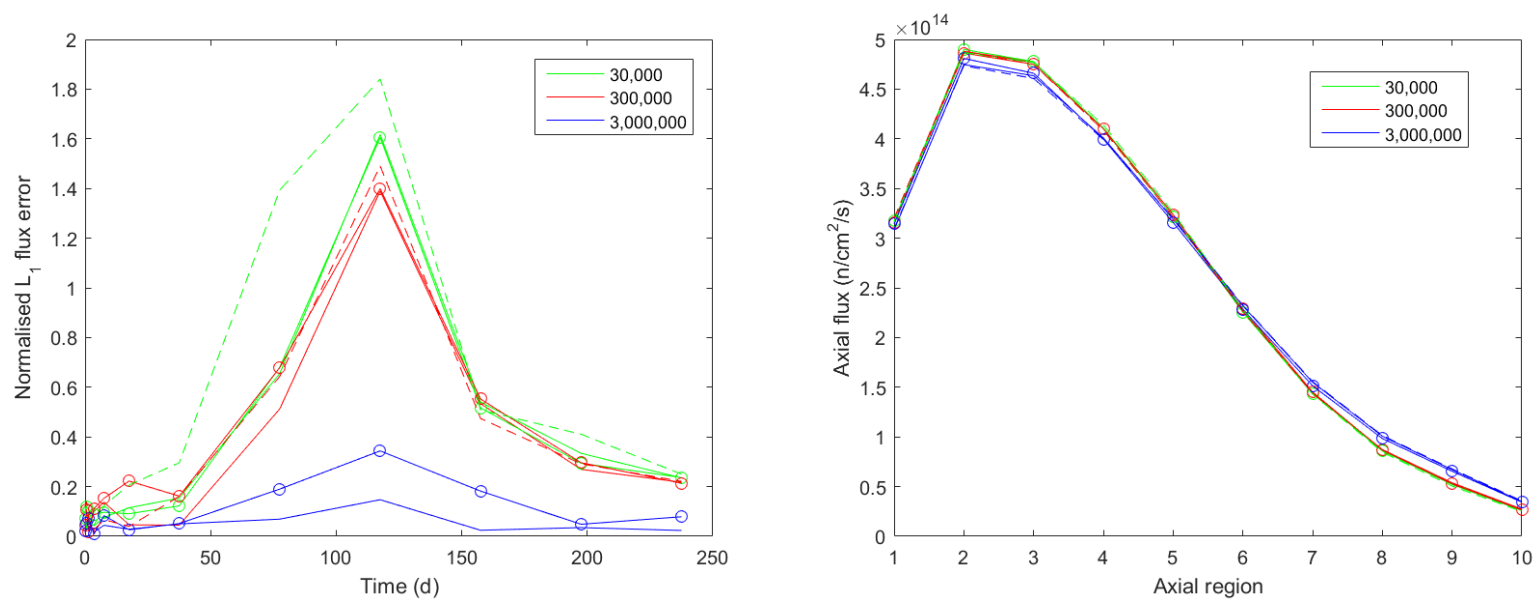

Fig. 13. Relative flux errors with time and final flux profiles when depleting a PWR pin with non-uniform coolant density using the PC scheme, with a 40 day maximum time-step, and varying the number of particles per generation while preserving the active histories

two underestimation of the neutron centre-of-mass oscillation (an indicator of clustering, like the first moment of Shannon entropy) when considering a full-core problem with vacuum boundaries. When neutrons families may be removed from the simulation through leakage, intuitively, one might imagine that the degree of correlation between particles would grow more rapidly due to the need to duplicate more surviving neutrons to ensure a constant population. However, although not presented in this work, in the authors' experience, even a reflected PWR pin may develop spurious oscillations with burn-up when using 300,000 particles per generation and relatively few generations, with a similar occurrence reported by Josey (2017).

The other explanation is that the sensitivity of depletion instability to errors in the eigenvector is not to be underestimated. Extending beyond Eqn. (5), Nowak et al. (2016) derive a number of expressions for the deviation of the neutron centre-of-mass. Using their Eqn. (35), for a $3.66 \mathrm{~m}$ fuel rod, simulating 300,000 or $3,000,000$ particles per generation will result in a fluctuation of $2.1 \mathrm{~cm}$ or $0.7 \mathrm{~cm}$ of the neutron centre-of-mass, respectively. As mentioned above, these are underestimates due to the neglect of leakage. However, it may be the case that the former perturbs the flux solution sufficiently to initiate a numerical xenon oscillation whereas the latter does not.

\section{Conclusions}

This work has demonstrated that many cases in which non-physical solutions occur during Monte Carlo/depletion coupling - that have previously been reported as 'numerical instability' or a problem of statistics - are actually due to neutron clustering. Simulating too few neutrons per generation, as previous studies have done, will generate spurious results. On the other hand, simulating enough neutrons to suppress clustering provides stable solutions, as demonstrated when depleting a PWR pin with uniform coolant density using 
the PC scheme - in contrast to previous findings, this will give the expected symmetric solution. Furthermore, even in cases which are less pathological and more realistic, i.e., where the coolant density profile resembles that in an operating reactor, the effects of neutron clustering still result in significantly differing numerical solutions. In all cases which use an insufficient number of particles to avoid clustering, the evolution of the neutronics/depletion system is stochastic, that is, prior to converging to a 'stable' non-physical state. Using excessively many inactive cycles also appears to drive burn-up instability, as would follow from the predictions of Sutton and Mittal (2017), albeit using too few particles to begin with appears to be a more dominant effect. Finally, this work has shown that different timestep lengths can result in different solutions, even when using many particles/generation. In spite of previous studies not accounting for clustering, this adds credence to claims of neutronics/depletion schemes being numerically unstable.

While this study has investigated one mechanism by which erroneous solutions are obtained, it has not rigorously considered the possibility of true numerical instability existing when coupling transport and depletion, driven by taking excessively large time-steps, as explored by Densmore et al. (2013). With or without clustering and noise, it may prove possible to estimate the stability of a high-fidelity neutronics/depletion problem and this will be considered in future work.

This work has focused on neutron clustering but, in principle, noise in the Monte Carlo solution attributable to other causes appears likely to result in the same detrimental effects. This was not a concern in the problems examined here given the relatively large number of active histories simulated such that reaction rate estimators were subject to a low degree of noise. Extending the current analysis to full-core problems where it is not uncommon to use on the order of 1-10 million particles/generation, one might find that clustering is not so dominant an effect as simply struggling to sufficiently converge local flux/reaction rate estimates - due to the many more regions in which reaction rates must be tallied, simulating many particles per generation as well as many more active cycles may be necessary to avoid non-physical behaviour.

Finally, if neutron clustering plays an important role in coupling Monte Carlo with depletion, it may be similarly important in other developments. For example, clustering may strongly impact source convergence acceleration techniques where an insufficient number of particles are used such that there may be a failure to converge to the fundamental eigenvector. For example, this might prove troublesome for or limit the usage of 'particle ramping' schemes, which simulate fewer particles at the beginning of the inactive cycles and increase particle numbers as the cycles progress, attempting to reduce wasted computational effort. Examples include those proposed by Dufek and Tuttelberg (2016) and Lund et al. (2017).

\section{Data availability statement}

To the best of the authors' knowledge, this paper and references herein contain all the data needed to reproduce and validate the results presented. 


\section{Acknowledgements}

This work was carried out with the financial support of the UK Engineering and Physical Sciences Research Council (EPSRC) through the Imperial College, University of Cambridge and Open University Centre for Doctoral Training in Nuclear Energy under grant $\mathrm{EP} / \mathrm{L} 015900 / 1$.

This work was performed using resources provided by the Cambridge Service for Data Driven Discovery (CSD3) operated by the University of Cambridge Research Computing Service (www.csd3.cam.ac.uk), provided by Dell EMC and Intel using Tier-2 funding from the Engineering and Physical Sciences Research Council (capital grant EP/P020259/1), and DiRAC funding from the Science and Technology Facilities Council (www.dirac.ac.uk).

\section{Declarations}

Declarations of interest: none.

\section{References}

Aufiero, M., Fratoni, M., 2017. A new approach to the stabilization and convergence acceleration in coupled Monte Carlo-CFD calculations: The Newton method via Monte Carlo perturbation theory. Nuclear Engineering and Technology 49, 1181-1188. doi:10.1016/J.NET.2017.08.005.

Bell, G., Glasstone, S., 1970. Nuclear Reactor Theory. US Atomic Energy Commission, Washington, DC.

Brissenden, R., Garlick, A., 1986. Biases in the estimation of Keff and its error by Monte Carlo methods. Annals of Nuclear Energy 13, 63-83. doi:10.1016/0306-4549(86)90095-2.

Brown, F.B., 2011. 'K-effective of the World' and Other Concerns for Monte Carlo Eigenvalue Calculations. Progress in Nuclear Science and Technology 2, 738-742. doi:10.15669/pnst.2.738.

Densmore, J.D., Gill, D.F., Griesheimer, D.P., 2013. Stability analysis of burnup calculations. Transactions of the American Nuclear Society 109, 695-698. doi:10.1007/s10967-012-2210-3.2.

Dufek, J., Hoogenboom, J.E., 2009. Numerical Stability of Existing Monte Carlo Burnup Codes in Cycle Calculations of Critical Reactors. Nuclear Science and Engineering 162, 307-311. doi:10.13182/NSE08$69 \mathrm{TN}$.

Dufek, J., Kotlyar, D., Shwageraus, E., 2013a. The stochastic implicit Euler method: A stable coupling scheme for Monte Carlo burnup calculations. Annals of Nuclear Energy 60, 295-300. doi:10.1016/j.anucene.2013.05.015.

Dufek, J., Kotlyar, D., Shwageraus, E., Leppänen, J., 2013b. Numerical stability of the predictor-corrector method in Monte Carlo burnup calculations of critical reactors. Annals of Nuclear Energy 56, 34-38. doi:10.1016/j.anucene.2013.01.018.

Dufek, J., Tuttelberg, K., 2016. Monte Carlo criticality calculations accelerated by a growing neutron population. Annals of Nuclear Energy 94, 16-21. doi:10.1016/J.ANUCENE.2016.02.015.

Dumonteil, E., Bruna, G., Malvagi, F., Onillon, A., Richet, Y., 2017. Clustering and traveling waves in the Monte Carlo criticality simulation of decoupled and confined media. Nuclear Engineering and Technology 49, 1157-1164. doi:10.1016/J.NET.2017.07.011.

Dumonteil, E., Diop, C.M., 2011. Biases and Statistical Errors in Monte Carlo Burnup Calculations: An Unbiased Stochastic Scheme to Solve Boltzmann/Bateman Coupled Equations. Nuclear Science and Engineering 167, 165-170. doi:10.13182/NSE09-100.

Dumonteil, E., Malvagi, F., Zoia, A., Mazzolo, A., Artusio, D., Dieudonné, C., De Mulatier, C., 2014. Particle clustering in Monte Carlo criticality simulations. Annals of Nuclear Energy 63, 612-618. doi:10.1016/J.ANUCENE.2013.09.008. 
Gill, D.F., Griesheimer, D.P., Aumiller, D.L., 2017. Numerical Methods in Coupled Monte Carlo and Thermal-Hydraulic Calculations. Nuclear Science and Engineering 185, 194-205. doi:10.13182/NSE16-3.

Griesheimer, D.P., Carpenter, D.C., Stedry, M.H., 2017. Practical techniques for large-scale Monte Carlo reactor depletion calculatons[sic]. Progress in Nuclear Energy 101, 409-423. doi:10.1016/j.pnucene.2017.05.018.

Herman, B.R., 2014. Monte Carlo and Thermal Hydraulic Coupling using Low-Order Nonlinear Diffusion Acceleration. Ph.D. thesis. Massachusetts Institute of Technology.

Isotalo, A., 2013. Computational Methods for Burnup Calculations with Monte Carlo Neutronics. Ph.D. thesis. Aalto University.

Isotalo, A., Aarnio, P., 2011. Substep methods for burnup calculations with Bateman solutions. Annals of Nuclear Energy 38, 2509-2514. doi:10.1016/j.anucene.2011.07.012.

Isotalo, A., Leppänen, J., Dufek, J., 2013. Preventing xenon oscillations in Monte Carlo burnup calculations by enforcing equilibrium xenon distribution. Annals of Nuclear Energy 60, 78-85. doi:10.1016/J.ANUCENE.2013.04.031.

Josey, C., 2017. Development and Analysis of High Order Neutron Transport-Depletion Coupling Algorithms. Ph.D. thesis. Massachusetts Institute of Technology.

Kang, C., Mosteller, R., 1983. Incorporation of a predictor-corrector depletion capability into the CELL-2 code. Transactions of the American Nuclear Society 45, 729-731.

Kȩpisty, G., Cetnar, J., 2015. Instabilities of Monte-Carlo burnup calculations for nuclear reactors-Demonstration and dependence from time step model. Nuclear Engineering and Design 286, 49-59. doi:10.1016/J.NUCENGDES.2015.01.023.

Kotlyar, D., Shwageraus, E., 2013. On the use of predictor-corrector method for coupled Monte Carlo burnup codes. Annals of Nuclear Energy 58, 228-237. doi:10.1016/j.anucene.2013.03.034.

Kotlyar, D., Shwageraus, E., 2014. Numerically stable Monte Carlo-burnup-thermal hydraulic coupling schemes. Annals of Nuclear Energy 63, 371-381. doi:10.1016/j.anucene.2013.08.016.

Kotlyar, D., Shwageraus, E., 2016. Stochastic semi-implicit substep method for coupled depletion MonteCarlo codes. Annals of Nuclear Energy 92, 52-60. doi:10.1016/j.anucene.2016.01.022.

Leppänen, J., Pusa, M., Viitanen, T., Valtavirta, V., Kaltiaisenaho, T., 2015. The Serpent Monte Carlo code: Status, development and applications in 2013. Annals of Nuclear Energy 82, $142-150$. doi:10.1016/j.anucene.2014.08.024.

Lund, A., Romano, P.K., Siegel, A., 2017. Accelerating Source Convergence in Monte Carlo Criticality Calculations Using a Particle Ramp-up Technique, in: M\&C, Jeju, Korea.

de Mulatier, C., Dumonteil, E., Rosso, A., Zoia, A., 2015. The critical catastrophe revisited. Journal of Statistical Mechanics: Theory and Experiment 2015, P08021. doi:10.1088/1742-5468/2015/08/P08021.

Nowak, M., Miao, J., Dumonteil, E., Forget, B., Onillon, A., Smith, K.S., Zoia, A., $2016 . \quad$ Monte Carlo power iteration: Entropy and spatial correlations. Annals of Nuclear Energy 94, 856-868. doi:10.1016/J.ANUCENE.2016.05.002.

Poston, D., Trellue, H., 1999. Development of a Fully-Automated Monte Carlo Burnup Code Monteburns. Technical Report LA-UR-99-42. Los Alamos National Laboratory. Los Alamos, New Mexico.

Pusa, M., 2013. Numerical Methods for Nuclear Fuel Burnup Calculations. Ph.D. thesis. VTT Technical Research Centre of Finland.

Stankovskiy, A., 2012. ALEPH2 - a general purpose Monte Carlo depletion code, in: PHYSOR, Knoxville, Tennessee.

Sutton, T.M., Mittal, A., 2017. Neutron clustering in Monte Carlo iterated-source calculations. Nuclear Engineering and Technology 49, 1211-1218. doi:10.1016/J.NET.2017.07.008.

Ueki, T., 2008. On-the-fly Judgments of Monte Carlo Fission Source Convergence. Transactions of the American Nuclear Society 11, 512-514.

Zoia, A., Dumonteil, E., Mazzolo, A., de Mulatier, C., Rosso, A., 2014. Clustering of branching Brownian motions in confined geometries. Physical Review E 90, 042118. doi:10.1103/PhysRevE.90.042118. 